\title{
Erratum
}

Mauro Zago'

\section{Time for a Comprehensive Ultrasound-Enhanced Trauma Management}

Eur J Trauma Emerg Surg 2009

DOI 10.1007/s00068-009-3004-3

There was an error in the description of the position of Lucas Greiner:

Please note the correct position:

[... ] Lucas Greiner, past Secretary of the European

Federation of Societies for Ultrasound in Medicine and Biology (EFSUMB).

Eur J Trauma Emerg Surg 2009;35:594

DOI 10.1007/s00068-009-3005-2

Published Online: November 16, 2009 